This item was submitted to Loughborough's Research Repository by the author.

Items in Figshare are protected by copyright, with all rights reserved, unless otherwise indicated.

\title{
Regular physical activity and insomnia: an international perspective
}

PLEASE CITE THE PUBLISHED VERSION

https://doi.org/10.1111/jsr.12745

PUBLISHER

Wiley ( E European Sleep Research Society

VERSION

AM (Accepted Manuscript)

\section{PUBLISHER STATEMENT}

This is the peer reviewed version of the following article: HARTESCU, I. and MORGAN, K., 2018. Regular physical activity and insomnia: an international perspective. Journal of Sleep Research, 28 (2), e12745, which has been published in final form at https://doi.org/10.1111/jsr.12745. This article may be used for noncommercial purposes in accordance with Wiley Terms and Conditions for Use of Self-Archived Versions.

\section{LICENCE}

CC BY-NC-ND 4.0

\section{REPOSITORY RECORD}

Hartescu, luliana, and Kevin Morgan. 2019. "Regular Physical Activity and Insomnia: An International Perspective". figshare. https://hdl.handle.net/2134/34169. 
TITLE: Regular physical activity and insomnia: an international perspective Iuliana Hartescu, $\mathrm{PhD}^{1}$

Kevin Morgan, $\mathrm{PhD}^{1}$

${ }^{1}$ Clinical Sleep Research Unit, School of Sport, Exercise and Health Sciences, Loughborough University, Loughborough LE11 3TU, UK.

Corresponding author: Dr luliana Hartescu (I.Hartescu@lboro.ac.uk)

Clinical Sleep Research Unit, School of Sport, Exercise and Health Sciences, Loughborough University, Loughborough LE11 3TU, UK.

Tel: 0044 (0)1509222760; Fax: +44 (0)1509 226301

\section{Financial support:}

Iuliana Hartescu and Kevin Morgan: The research reported here was supported by Sealy UK.

There was no off-label or investigational use of a therapeutic product in this study. None of the authors has any conflict of interests to report.

Author contributorship: Iuliana Hartescu and Kevin Morgan both contributed to all sections of the paper.

Words: 3613 words

References: 28 


\section{ABSTRACT}

Very low, and very high levels of regular physical activity have been associated with degraded sleep quality. Cross-national variations in habitual physical activity levels may contribute to cross-national differences in insomnia prevalence. The present study assesses and compares the extent to which weekly durations of moderate intensity physical activity contribute to insomnia risk. Demographic, sleep, physical activity and general health profiles were obtained from a convenience sample of 9238 adults drawn from 5 countries (South Africa, Australia, China, South Korea and the UK) using social media. Insomnia prevalence ranged from 4.1\% (China) to 14.8\% (UK). In logistic regression adjusted only for age and gender, the lowest level of activity was associated with significant insomnia risk (OR $=1.37(95 \% \mathrm{Cl}=1.05-$ $1.79 ; p<0.05)$. When adjusted for all covariates except country, only the highest level of physical activity (>300 min/week) was associated with significantly increased insomnia risk $(\mathrm{OR}=1.30(95 \% \mathrm{Cl}=1.03-2.51 ; \mathrm{p}<0.05)$. Risk associated with high activity remained after the addition of 'country' to the model $(\mathrm{OR}=1.31(95 \% \mathrm{Cl}$ $=1.02-1.69 ; p<0.05)$. Across all models, female gender, low rated health, low education, and older age consistently increased insomnia risk. These cross-national data indicate that extremes of inactivity/activity can significantly influence insomnia risk independent of country. Insomnia risk associated with very low levels of activity may be mediated by poorer health and disadvantageous social status. However, while very high levels of activity increase insomnia risk independent of health and demographic factors, they may also confound with personally and occupationally demanding lifestyles. 


\section{INTRODUCTION}

While Spielman, Caruso \& Glovinsky's (1987) '3-factor' model provides a framework for understanding the interaction of acute and chronic processes in the etiology of insomnia, epidemiological research has consistently identified personal attributes associated with elevated insomnia risk, including female gender, older age, degraded health, lower socio-economic status (Ohayon, 2002) and, more recently, physical inactivity (e.g. Kim et al, 2009). Low levels of physical activity have been reported as significant risk factors for both prevalent and incident insomnia (Janson et al, 2001; Morgan, 2003; Kim et al, 2009; Sporndly-Nees, Asenlof \& Lindberg, 2017; Chen, Steptoe, Chen \& Lin, 2017). Typically, however, studies to date have focused either exclusively on leisure activities and/or report only dichotomized (higher versus lower) activity levels. It remains unclear just how much activity (whether occupational, leisure, or routine activities of daily living) is sufficient to offset sleep-risk. In controlled trials with those reporting insomnia symptoms, for example, significant improvements in sleep quality have been achieved from programs requiring 100 min/week (Reid et al., 2010) through 150 min/week (Passos et al, 2012) of aerobic activity, or $210 \mathrm{~min} /$ week of moderate-intensity endurance exercise (King et al., 2008). Hartescu , Morgan and Stevinson (2015), have also shown that relatively modest levels of activity maintained at or above the World Health Organization (WHO, 2010) recommended minimum threshold (150 mins/week of moderate intensity activity) can significantly mitigate symptom severity among sedentary people with insomnia. Whether levels of activity below this threshold confer protective benefits is unknown.

Evidence from both epidemiological and experimental studies also indicates that the relationship between activity levels and improved wellbeing and sleep quality is 
complex. Among predominantly middle-class college graduates in the United States, Galper et al (2006) report a significant dose-response relationship between higher levels of self-reported activity and improved psychological wellbeing. However, survey findings from random samples in 28 European countries, showed that while regular moderate-intensity activity (above WHO guidelines) was associated with improved subjective wellbeing, regular vigorous-activity was associated with significantly degraded wellbeing (Wicker and Frick, 2015). Consistent with this latter finding, a systematic review of the sleep-sport literature (Gupta, Morgan and Gilchrist, 2017) concluded that the prevalence of insomnia symptoms was consistently elevated for elite athletes across a range of sports. Given this literature, there would seem to be clear methodological benefits, when evaluating activityinsomnia relationships, in using a range of activity categories which capture (and isolate) both the very inactive and the very active.

Despite the potential benefits for general health of increased moderate activity levels (Lee et al, 2012), the prevalence of 'insufficient physical activity' (i.e. activity falling below the WHO 150 mins/week threshold) continues to rise internationally, though showing considerable cross-national variability (WHO, 2010). Cross-national levels of insomnia symptoms also show considerable variations. In a 10-country survey Soldatos, Allaert, Ohta, \& Dikeos (2005), for example, reported an overall pointprevalence rate for insomnia (meeting DSM IV criteria) of $12 \%$, with country-specific rates ranging from 5\% (Germany) to 32\% (Brazil). Similarly, Stranges, Tigbe, Gomez-Olive, Thorogood \& Kandala (2012) in a study of 8 low-income countries, report an overall prevalence of insomnia symptoms of $16.6 \%$, but country specific rates ranging from 3.9\% (Purworejo, Indonesia) to over 40.0\% (Matlab, Bangladesh). 
While cross-national variations in sleep experience may reflect cultural perceptions and beliefs, they may also reflect underlying variations in those factors already identified as sleep quality risks. However, this possibility is under-explored; few studies have simultaneously collected and compared cross-national data on sleep quality and lifestyle factors. The explicit aim of the present study was to assess relationships between physical activity levels and sleep quality using a range of activity categories defined with reference to WHO (2010) guidelines, and analyzed in the context of demographic and health variables known to influence insomnia risk. The 5-Nation Sleep Survey was designed to simultaneously collect data in five countries on four continents. In sampling countries the aim was to recruit participants in both developed and emerging economies, and from Northern and Southern hemispheres, in order to provide geographic and socio-demographic diversity. Analyses addressed three research questions:

1. In unadjusted analyses, is insomnia risk significantly associated with the reported daily/weekly duration of (at least) moderate intensity physical activity?

2. Is insomnia risk significantly associated with the weekly duration of (at least) moderate intensity physical activity when appropriate confounders are controlled? and

3. If present in adjusted models, is the relationship between weekly physical activity and insomnia risk influenced by the participants' country of origin.

All research questions adopted the same null hypothesis: levels of moderate intensity physical activity are not significantly related to insomnia risk.

\section{METHOD}




\section{Study design and participants}

The online questionnaire contained 48 items covering aspects of demographic status, health, sleep behavior, sleep quality, and lifestyle. All questions were first written in English before being translated into Chinese (simplified) and Korean (Hangul). The English and Korean versions were mounted on Survey Monkey; the Chinese version was mounted on a commercial cloud-based system through eqxiu.com. The completed questionnaires were then distributed to the target countries (South Africa, Australia, China, South Korea and the UK) through a single agent specializing in digital public relations campaigns (Prohibition PR, Leeds, UK: https://prohibitionpr.co.uk/). Within each target country partner agencies distributed links to the questionnaire through social media and websites. The questionnaire was opened on July $15^{\text {th }} 2016$ and closed on August $31^{\text {st }} 2016$. In all countries the survey aims were explained in an opening header requiring active consent (a checkbox) before proceeding.

\section{Measures}

Demographic items covered age, gender, education level and employment status and country of origin. Education level was coded using the Eurostat (2016) aggregation of the International Standard Classification of Education (ISCE) categories (UNESCO, 2012): Low Education (less than primary, primary and lower secondary education); Medium Education (upper secondary and post-secondary non-tertiary education); and High Education (tertiary education). Lifestyle items addressed pre-bedtime routines, caffeine, alcohol, and cigarette use. General health was assessed using the global item "How would you rate your present health" with the response options: well above average; above average; average; below average; 
and well below average. A further item captured ongoing conditions. All participants completed questions addressing DSM-5 criteria for insomnia: "In the past 3 months have you had problems with getting to sleep?; In the past 3 months have you had problems with staying asleep (or waking too early in the morning)?" These items were followed by an inventory of daytime consequences: "In the past 3 months have any of the following resulted from lack of sleep?", with 8 options covering productivity, mood stability, accidents, absenteeism, punctuality, low mood, sleepiness and fatigue. Additional sleep items included a global rating of sleep quality (very good to very poor) and sleep medication use (yes/no) in the previous 3 months. To assess subjective sleep adequacy participants were asked to estimate: 1) the number of hours typically slept "during a normal working week"; and, later in the questionnaire, 2) the number of hours sleep generally needed "...to function effectively the next day".

Physical activity was assessed from two questions on: 1) the typical number of days per week during which participants engaged in moderate intensity exercise (e.g. walking at a moderate pace) for at least 10 continuous minutes; and 2) the amount of time typically spent on continuous moderate activity on these active days. Response formats offered a narrow range of days (<1; $1-2 ; 3-4 ; 5-6$; and 7$)$ and durations (10$20 \mathrm{~min}$; $21-30 \mathrm{~min}$; 31-60 min; 61-120-min; >120 min) respectively. From these items weekly durations of at least moderate intensity physical activity were estimated as the product of days and durations (using range mid points from both measures. For example, a participant reporting 21-30 min of activity on 3-4 days per week would score $(24.5 \times 3.5) \mathrm{min} /$ week.

\section{Data Analyses}


Data collected in each country were sent to Loughborough University, where they were collated and analyzed according to an agreed protocol. Estimated weekly durations of moderate intensity physical activity were divided into categories above and below WHO international physical activity guidelines for health $(\geq 150$ minutes/week) as follows: <10 min/week; 10-75 min/week; 76-149 min week; 150$300 \mathrm{~min} /$ week; and $>300 \mathrm{~min} /$ week. Those reporting insomnia symptoms $\geq 3$ times/week for the previous 3 months, adequate sleep opportunity, daytime consequences, and no concomitant mental or physical health problem were categorized as an insomnia 'case'. Prevalence estimates and descriptive statistics were then computed for the sample overall, and for each country separately. To address the study's first research question, the unadjusted association between insomnia prevalence and minutes spent in moderate intensity physical activity per typical day, and per week were assessed in contingency tables using $X^{2}$. Because international physical activity guidelines (WHO, 2010) are expressed as minutes per week, the remaining analyses focused on weekly activity levels. Three logistic regression models were then separately fitted to address research questions 2 and 3. Model 1, a partially adjusted model, included insomnia case/noncase as the dependent variable, and minutes per week spent in (at least) moderate intensity physical activity, age and gender as covariates. In Model 2, subjectively rated health, sleep medication use in the previous 3 months, smoking status (smoker/nonsmoker), alcohol consumption (<21 or $>21$ units/week), caffeinated drink consumption within 1 hour of going to bed (yes/no), educational level (high, medium, or low), and employment status (formally employed/not formally employed) were added to Model 1 covariates. Finally, in Model 3, country of residence was added to the Model 2 covariates. In addition to main effects, the following interaction terms 
were initially added to the models: physical activity level $x$ gender to Models 1,2 and 3; physical activity level x employment status to Models 2 and 3; and country $x$ physical activity level, country x employment status, country $x$ gender, and country $x$ age to Model 3. All data were analyzed using IBM SPSS 23. Statistical tests were two tailed with alpha set at the $p<05$ level.

\section{RESULTS}

\section{Sample characteristics}

A total of 9238 participants completed the questionnaire; all were aged 18 and over and were drawn from five countries (and four continents): South Africa, Australia, China, South Korea and the UK. The geographic distribution and demographic characteristics of the sample are shown in Table 1. The survey was mostly completed by people in formal employment (69.3\%), and by women (75.9\%). Of those not formally employed, most were retired (37\%), full-time caregivers (35\%) or students (10\%). In each country most participants were aged under 55, with the proportion of those $55+$ ranging from $32 \%$ (UK) to $1.4 \%$ (China). Overall, the sample was well educated, with only a small minority in each country reporting a maximum educational level lower than upper secondary. Health-profiles for each national sample are shown in Table 2. Levels of smoking and alcohol consumption (greater than 21 units/week) were generally low, though both behaviors were highest among men, and particularly high among males in China and South Korea. In each country, most participants (over $70-80 \%$ in South Africa, Australia, China and the UK) rated their health as either 'average' or 'above average'. These ratings were lowest in South Korea. 


\section{Sleep health and physical activity}

Sleep Quality

Over $50 \%$ of participants in all countries rated their sleep quality as "Good" (Table 2), with overall levels of positive sleep quality ratings ("Good + "Very Good) ranging from $56.5 \%$ (UK women) to $77.2 \%$ (South Korean women). For both women and men in all countries the average reported duration of sleep typically obtained was less than that needed to "...function effectively the next day" (Table 2), with differences between these two values ranging from $0.2 \mathrm{hr}$ (Australian and UK men) to $1.4 \mathrm{hr}$ (South Korean men). Insomnia prevalence rates for South Africa, Australia, and the UK fell within the range 10-15\%. For China and South Korea, however, insomnia prevalence was substantially lower ( $4.1 \%$ and $6.5 \%$ respectively; Table 3$)$. For the sample overall there was a significant association between gender and sleep quality, with $13.0 \%$ of women and $6.5 \%$ of men meeting the survey criteria for insomnia (Table 3); $(x 2(1)=71.95, p<0.001)$. In all countries insomnia prevalence and sleep medication use was highest among women.

Physical activity

Prevalence rates of physical activity within each the study categories are shown in Table 3. For all countries, the modal category was $10-75$ minutes of at least moderate intensity physical activity per week, with levels of very low activity $(<10$ continuous minutes/day) ranging from 6\% (China) to 9.5\% (South Korea). With data from all countries aggregated, there was a significant association between reported activity levels and gender, with a greater proportion of men in the higher categories, 
and a greater proportion of women in the lower categories $(x 2(4)=101.5, p<0.001)$. South Africa and South Korea reported the highest levels of low activity (defined as those levels less than 150 mins/week of moderate intensity physical activity), with $79 \%$ and $69 \%$ of participants respectively reporting weekly levels $<150$ min/week.

\section{Insomnia and physical activity}

Significant bivariate associations were present between insomnia prevalence and reported minutes of moderate intensity activity per typical active day $\left(X^{2}(4,9238)=\right.$ $14.77, \mathrm{p}<.005))$ and reported minutes of moderate intensity physical activity per week $\left(X^{2}(4,9238)=14.90, p<.01\right)$. In both cases, insomnia prevalence was elevated for the lowest and the highest levels of activity (see Figures 1 and 2).

Assumptions for logistic regression were checked and met. Odds ratios and 95\% confidence intervals for all the models are shown in Table 4. In Model 1, adjusted only for age and gender, insomnia risk was significantly elevated only for the lowest level of physical activity $(\mathrm{OR}=1.37(95 \% \mathrm{Cl}=1.05-1.79 ; \mathrm{p}=.015))$. Results from partially adjusted Model 2 are also shown in Table 4. With additional adjustment for subjectively rated health, sleep medication use, smoking status, alcohol consumption, caffeine use before bedtime, educational level and employment status, the highest level of physical activity was associated with significant insomnia risk $(\mathrm{OR}=1.30(95 \% \mathrm{Cl}=1.01-1.65 ; \mathrm{p}=.045))$. The addition, in Model 3, of 'country' (Table 4) did not substantially alter the high activity - insomnia risk relationship. None of the interaction terms was significant (Table 4): physical activity level $X$ gender in Models 1, 2 and 3; physical activity level x employment status in Models 2 
and 3; and country $x$ physical activity level, country $x$ employment status, country $\mathrm{x}$ gender, and country $x$ age in Model 3. 


\section{DISCUSSION}

The present study achieved its methodological aim of collecting sleep health and physical activity data in 5 countries over the same time period. Completion rates among those who opened the questionnaire was high (over 90\%). Given that participant recruitment was effected almost exclusively through shared information on digital platforms, the demographic structure of the resulting convenience sample is consistent with research showing the greater use of online social networks by women (e.g. Kimbrough, Guadagno, Muscanell, and Dill, 2013), and younger people (e.g. Vosner, Bobek, Kokol, and Krecic, 2016). However, since the present analyses were designed to explore relationships among variables in models adjusted for age and gender, no attempt was made to weight the present samples back to national population norms. In the event, both insomnia prevalence (Table 3), and the contribution of demographic factors to insomnia risk (Table 4), are all in line with previous epidemiological findings. Overall insomnia rates for South Africa, Australia and the UK reported here (10-15\%; Table 3) fall within the ranges reported in the reviews of Ohayon (2002) and Morgan (2012) covering Africa, Australasia and Europe. The comparatively lower insomnia prevalence rates for Asian countries (47\%; Table 3), while possibly influenced by sample structure, are consistent with those reported for South Korea by Ohayon and Hong (2002), and the range of insomnia rates for China reviewed by Cao, Wang, Zhong, Zhang, Ungvari, Ng et al. (2017). In addition to prevalence levels, the multivariate models (Table 4) consistently reflect the significantly increased insomnia risk associated with older age, female gender, lower self-rated health, and lower levels of education noted in (and expected from) previous reports (e.g. Ohayon, 2002). We are confident, 
therefore, that the present sampling strategy appropriately identified symptomatic sub-samples reflecting the clinical dynamics characteristic of insomnia cases.

Within this predominantly working-age sample, 3 outcomes show consistency across both countries and genders: the majority of participants in all countries expressed satisfaction with their typical sleep quality; the average sleep typically obtained each night was less than that considered necessary for effective daytime functioning; and most participants report physical activity levels below WHO recommended minimum threshold (150 mins/week of moderate intensity activity). Differences between subjectively 'necessary' and 'obtained' sleep durations need to be interpreted in context. While an apparent sleep 'debt' may indicate a global pressure to prioritize waking behavior over sleep, it may also be the case that this finding is influenced, to an unknown degree, by unrealistic expectations of 'normal' sleep. South Korea, for example, showed the greatest difference between subjective sleep need and sleep obtained $(1.1 \mathrm{hr})$. It is clear from Table 2, however, that while 'obtained' sleep durations were similar across countries, South Korean women and men reported both the highest average values for subjective sleep need and the highest levels of sleep satisfaction.

The high prevalence rates of low physical activity reported for each participating country (Table 3) accord with what has been described as a "pandemic of physical inactivity" (Kohl, Craig, Lambert, Inoue, Alkandari, Leetongin, et al. 2012). Nevertheless, the present findings indicate both positive and negative associations between activity levels and sleep quality. In the unadjusted analyses (Figures 1 and 2), insomnia risk was highest for very low and very high levels of reported daily and weekly durations of (at least) moderate intensity physical activity. In the logistic regression analyses the lowest level of weekly activity remained a significant risk for 
insomnia after adjustment for age and gender in the Model 1 . However, after adjustment for a range of confounders, Model 2 showed no significant risk for the lowest levels of activity, but did show significant risk for the highest level of activity. A reasonable interpretation of these findings is that the lowest levels of physical activity in Model 1 served as a proxy for health status and other contextual variables; hence, the control of these variables in Model 2 rendered the low-activity - insomnia relationship non-significant for this sample. Of interest, however, is that in the fully controlled model, the highest level of physical activity (>300 minutes/week) emerged as a risk for insomnia $(\mathrm{OR}=1.30(95 \% \mathrm{Cl}=1.03-2.51 ; \mathrm{p}<0.05)$. That the overall pattern of insomnia risk associated with activity, health and demographic variables was not substantially changed by the addition of the 'country of origin' variable (Model 3) strongly suggests that these findings are internationally robust. Similar to other epidemiological studies (e.g. Galper et al, 2006 who classified $12.5 \%$ of their participants as 'highly active'), only a minority of individuals in the present study (12.2\%; see Table 3) reported the highest levels of physical activity (>300 minutes/week).

Two broad conclusions can be drawn from the present findings. First, alongside those variables more usually associated with insomnia symptoms, physical activity remains an important consideration when assessing insomnia risk. Second, at levels consistent with cardiovascular and metabolic benefits (i.e. >300 min/week in the present study), regular physical activity may, under some circumstances, be associated with an increased risk of insomnia. While the evidence supporting this latter point clearly aligns with Wicker and Frick's (2015) finding of degraded subjective wellbeing among those reporting higher levels and intensities of regular activity, such findings should be interpreted with considerable caution. A limitation of 
the present study is that it did not explore (and could not control for) the nature of the regular activities reported by participants. The online questionnaire audited all relevant activities, whether occupational or recreational, or reflecting preference or obligation. A plausible explanation for the current findings, therefore, is that in this predominantly younger working-age sample, some drivers of higher activity also served to degrade sleep quality. For example longer working hours (Alfonso et al, 2017), demanding occupational labour (Smagula et al, 2016), protracted caregiving (McCurry et al, 2015), parenting (Hagen et al, 2013) and competitive athletic training (Gupta and Morgan, 2016) can all be expected to elevate daytime activity levels, but have all been significantly linked to increased insomnia risk. This explanation has relevance both for the public-health implications of the present findings, and research methodology. Regarding public health, the present results do not allow a conclusion that higher levels of activity per se increase insomnia symptoms. And regarding methodology, future studies should recognise the importance of identifying the ecological context, as well as quantifying levels of regular physical activity. Finally, it should also be acknowledged that the present data collection method failed to discriminate between moderate and vigorous activity, adopting instead the catchall "at least moderate intensity" definition. This intentional strategy aimed to keep the questionnaire accessible and understandable, but necessarily sacrificed detail.

\section{CONCLUSION}

Using DSM-5 criteria the present study shows a consistent and interpretable pattern of insomnia prevalence and insomnia risk in 5 countries assessed within the same time-frame. While the more established health and demographic factors previously associated with insomnia risk appear active in all countries, the addition of regular physical activity adds explanatory power in multivariate models. In particular, the 
present findings identify associations between both very high, and very low levels of physical activity and sleep quality. As the emphasis on global public health benefits associated with regular physical activity increases, the need to include information on both levels and drivers of activity in epidemiologic studies of sleep is reinforced by the present findings.

\section{ACKNOWLEDGEMENTS}

We thank Will Ockenden and his colleagues at Prohibition PR Ltd for coordinating the distribution of the questionnaire. This research was supported by Sealy UK. 


\section{REFERENCES}

Afonso, P., Fonseca, M., \& Pires, J. F. (2017). Impact of working hours on sleep and mental health. Occupational Medicine-Oxford, 67(5), 377-382.

Cao, X.L., Wang, S.B., Zhong, B.L., Zhang, L., Ungvari, G.S., Ng, C,H,, Li, L,, Chiu, H.F., Lok, G.K., Lu, J.P., Jia, F.J. and Xiang, Y.T. The prevalence of insomnia in the general population in China: A meta-analysis. PLoS One., 2017, 12: e0170772.

Chen, L.J,. Steptoe, A., Chen, Y.H., Ku, P.W. and Lin, C.H. Physical activity, smoking, and the incidence of clinically diagnosed insomnia. Sleep Med., 2017, 30: 189-194.

Galper, D. I., Trivedi, M. H., Barlow, C. E., Dunn, A. L., \& Kampert, J. B. (2006). Inverse association between physical inactivity and mental health in men and women. Med Sci Sports Exerc., 38(1), 173-178.

Gupta, L., Morgan, K. and Gilchrist, S. Does Elite Sport Degrade Sleep Quality? A Systematic Review. Sports Med., 2017, 47: 1317-1333.

Hagen, E. W., Mirer, A. G., Palta, M., \& Peppard, P. E. (2013). The Sleep-Time Cost of Parenting: Sleep Duration and Sleepiness Among Employed Parents in the Wisconsin Sleep Cohort Study. Am J Epidemiol., 177(5), 394-401.

Hartescu, I., Morgan, K. and Stevinson, C.D. Increased physical activity improves sleep and mood outcomes in inactive people with insomnia: a randomized controlled trial. J Sleep Res., 2015, 24: 526-34.

Kim, J.M., Stewart, R., Kim, S.W., Yang, S.J., Shin, I.S. and Yoon, J.S. Insomnia, depression, and physical disorders in late life: a 2-year longitudinal community study in Koreans. Sleep., 2009, 32: 1221-8.

Kimbrough, A. M., Guadagno, R. E., Muscanell, N. L., and Dill, J.. Gender differences in mediated communication: Women connect more than do men. Comput Human Behav., 2013, 29: 896-900.

King, A. C., Oman, R. F., Brassington, G. S., Bliwise, D. L. and Haskell, W. L. Effects of moderate-intensity exercise on polysomnographic and subjective sleep quality in older adults with mild to moderate sleep complaints. J. Gerontol. A Biol. Sci. Med. Sci., 2008, 63: 997-1004.

Kohl 3rd, H. W., Craig, C. L., Lambert, E. V., Inoue, S., Alkandari, J. R., Leetongin, G., ... \& Lancet Physical Activity Series Working Group. (2012). The pandemic of physical inactivity: global action for public health. Lancet, 2012, 380: 294-305.

Lee, I. M., Shiroma, E. J., Lobelo, F., Puska, P., Blair, S. N., Katzmarzyk, P. T., \& Lancet Physical Activity Series Working Group. Effect of physical inactivity on major non-communicable diseases worldwide: an analysis of burden of disease and life expectancy. Lancet, 2012, 380: 219-229. 
McCurry, S. M., Song, Y., \& Martin, J. L. (2015). Sleep in caregivers: what we know and what we need to learn. Curr Opin Psychiatry., 28(6), 497-503.

Morgan, K. The epidemiology of sleep. In: C. M. Morin, \& C. A. Espie (eds), The oxford handbook of sleep and sleep disorders. OUP, New York, 2012: 303.

Ohayon, M. M. Epidemiology of insomnia: what we know and what we still need to learn. Sleep med rev., 2002, 6: 97-111.

Ohayon, M. M., \& Hong, S. C. Prevalence of insomnia and associated factors in South Korea. J Psychosom Res., 2002, 5: 593-600.

Passos, G. S., Poyares, D., Santana, M. G., D'Aurea, C. V. R., Youngstedt, S. D., Tufik, S., et al. (2011). Effects of moderate aerobic exercise training on chronic primary insomnia. Sleep Med., 12(10), 1018-1027.

Reid, K. J., Baron, K. G., Lu, B., Naylor, E., Wolfe, L. and Zee, P. C. Aerobic exercise improves self-reported sleep and quality of life in older adults with insomnia. Sleep Med., 2010, 11: 934-940.

Smagula, S. F., Stone, K. L., Fabio, A., \& Cauley, J. A. (2016). Risk factors for sleep disturbances in older adults: Evidence from prospective studies. Sleep Med Rev. , 25, 21-30.

Soldatos, C. R., Allaert, F. A., Ohta, T. and Dikeos, D. G. How do individuals sleep around the world? Results from a single-day survey in ten countries. Sleep Med., 2005, 6: 5-13.

Spielman, A. J., Caruso, L. S. and Glovinsky, P. B. A behavioral perspective on insomnia treatment. Psychiatr Clin North Am., 1987, 10: 541-553.

Sporndly-Nees, S., Asenlof, P. and Lindberg, E. High or increasing levels of physical activity protect women from future insomnia. Sleep Med., 2017, 32: 22-27.

Stranges, S., Tigbe, W., Gómez-Olivé, F. X., Thorogood, M. and Kandala, N. B. Sleep problems: an emerging global epidemic? Findings from the INDEPTH WHOSAGE study among more than 40,000 older adults from 8 countries across Africa and Asia. Sleep., 2012, 35: 1173-1181.

UNESCO Institute for Statistics (UIS). International Standard Classification of Education: ISCED 2011. UIS, Montreal, Quebec, 2012.

Vosner, H. B., Bobek, S., Kokol, P. and Krecic, M. J. (2016). Attitudes of active older Internet users towards online social networking Comput Human Behav., 2016, 55: 230-241.

WHO. Global recommendations on physical activity for health. Geneva: World Health Organization; 2010. 
Wicker, P. and Frick, B. (2015). The relationship between intensity and duration of physical activity and subjective well-being. Eur J Public Health., 2015, 25: 868-872. 
TABLE 1

Characteristics of participants in the 5-Nation Sleep Survey

\begin{tabular}{|c|c|c|c|c|c|c|}
\hline Categories & South Africa & Australia & China & $\begin{array}{l}\text { South } \\
\text { Korea }\end{array}$ & UK & Total \\
\hline $\begin{array}{l}\text { Total Participants } \\
\text { in each country }\end{array}$ & 2258 & 2052 & 1596 & 494 & 2838 & 9238 \\
\hline \multicolumn{7}{|l|}{ Gender: N (\%) } \\
\hline Female & 2058 (91.1) & $1511(73.6)$ & $853(53.4)$ & $333(67.4)$ & $2256(79.5)$ & $7011(75.9)$ \\
\hline Male & 200 (8.9) & $541(26.4)$ & $743(46.6)$ & $161(32.6)$ & $582(20.5)$ & $2227(24.1)$ \\
\hline \multicolumn{7}{|c|}{ Age group (years): N (\%) } \\
\hline $18-34$ & $609(27.0)$ & $757(36.9)$ & $998(62.5)$ & $184(37.2)$ & $616(21.7)$ & $3164(34.2)$ \\
\hline $35-54$ & $1275(56.5)$ & $904(44.1)$ & $575(36.0)$ & $265(53.6)$ & $1310(46.2)$ & $4329(46.9)$ \\
\hline $55-64$ & $289(12.8)$ & $273(13.3)$ & $21(1.3)$ & $36(7.3)$ & $636(22.4)$ & $1255(13.6)$ \\
\hline $65+$ & $85(3.8)$ & $118(5.8)$ & $2(0.1)$ & $9(1.8)$ & $276(9.7)$ & $490(5.5)$ \\
\hline \multicolumn{7}{|c|}{ Education Level: N (\%) } \\
\hline $\begin{array}{l}\text { Low: Less than } \\
\text { primary, primary } \\
\text { and lower } \\
\text { secondary }\end{array}$ & $124(5.5)$ & $111(5.4)$ & $42(2.6)$ & $3(0.6)$ & $85(3.0)$ & $365(4.0)$ \\
\hline $\begin{array}{l}\text { Medium: Upper } \\
\text { secondary and } \\
\text { post-secondary } \\
\text { non-tertiary }\end{array}$ & $1748(77.4)$ & $1181(57.6)$ & $979(61.3)$ & $180(36.4)$ & $1886(66.5)$ & $5974(64.7)$ \\
\hline High: Tertiary & $386(17.1)$ & $760(37.0)$ & $575(36.0)$ & $311(63.0)$ & $867(30.5)$ & $2899(31.4)$ \\
\hline \multicolumn{7}{|c|}{ Employment status: N (\%) } \\
\hline Formally employed & 1935 (85.7) & $1419(69.2)$ & $977(61.2)$ & $384(77.7)$ & $1686(59.4)$ & $6401(69.3)$ \\
\hline $\begin{array}{l}\text { Not formally } \\
\text { employed }\end{array}$ & $323(14.3)$ & $633(30.8)$ & $619(38.8)$ & $110(22.3)$ & $1152(40.6)$ & $2837(30.7)$ \\
\hline
\end{tabular}


Table 2

Health behaviours and subjective health among survey participants in 5 countries

\begin{tabular}{|c|c|c|c|c|c|c|c|c|c|c|c|c|c|}
\hline \multirow{2}{*}{\multicolumn{2}{|c|}{ Variable }} & \multicolumn{2}{|c|}{ South Africa } & \multicolumn{2}{|c|}{ Australia } & \multicolumn{2}{|c|}{ China } & \multicolumn{2}{|c|}{ South Korea } & \multicolumn{2}{|c|}{ UK } & \multicolumn{2}{|c|}{ Total } \\
\hline & & Women & Men & Women & Men & Women & Men & Women & Men & Women & Men & Women & Men \\
\hline \multicolumn{2}{|l|}{ Total N } & 2058 & 200 & 1511 & 541 & 853 & 743 & 333 & 161 & 2256 & 582 & 7011 & 2227 \\
\hline \multicolumn{2}{|c|}{ Cigarette smoker (\%) } & 17.5 & 18.5 & 8.7 & 9.4 & 2.6 & 33.2 & 3.9 & 32.3 & 16.1 & 9.6 & 12.4 & 19.9 \\
\hline \multicolumn{2}{|c|}{$\begin{array}{l}\text { Consuming }>21 \text { units } \\
\text { alcohol/week }(\%)\end{array}$} & 4.9 & 10.5 & 4.8 & 14.4 & 3.5 & 17.0 & 9.0 & 24.8 & 11.0 & 22.3 & 6.9 & 17.7 \\
\hline \multicolumn{2}{|c|}{$\begin{array}{l}\text { Consuming caffeinated } \\
\text { drinks within } 1 \text { hour of } \\
\text { bedtime }(\%)\end{array}$} & 18.1 & 24.0 & 7.4 & 5.3 & 20.6 & 14.0 & 4.3 & 5.9 & 14.4 & 13.0 & 14.0 & 11.7 \\
\hline \multicolumn{2}{|c|}{$\begin{array}{l}\text { Consuming prescription } \\
\text { sleep medication in } \\
\text { previous } 3 \text { months (\%) }\end{array}$} & 8.7 & 5.7 & 5.0 & 3.4 & 5.5 & 3.9 & 3.7 & 1.1 & 5.8 & 4.6 & 6.4 & 3.9 \\
\hline \multirow{2}{*}{$\begin{array}{l}\text { Self-Rated } \\
\text { health (\%): }\end{array}$} & $\begin{array}{l}\text { "Above } \\
\text { Average" }\end{array}$ & 45.3 & 51.5 & 45.2 & 51.2 & 41.1 & 43.5 & 13.5 & 21.7 & 39.1 & 45.9 & 41.3 & 45.1 \\
\hline & "Average" & 36.3 & 31.5 & 37.5 & 35.5 & 47.9 & 44.7 & 42.9 & 47.8 & 31.5 & 33.0 & 36.7 & 38.4 \\
\hline
\end{tabular}




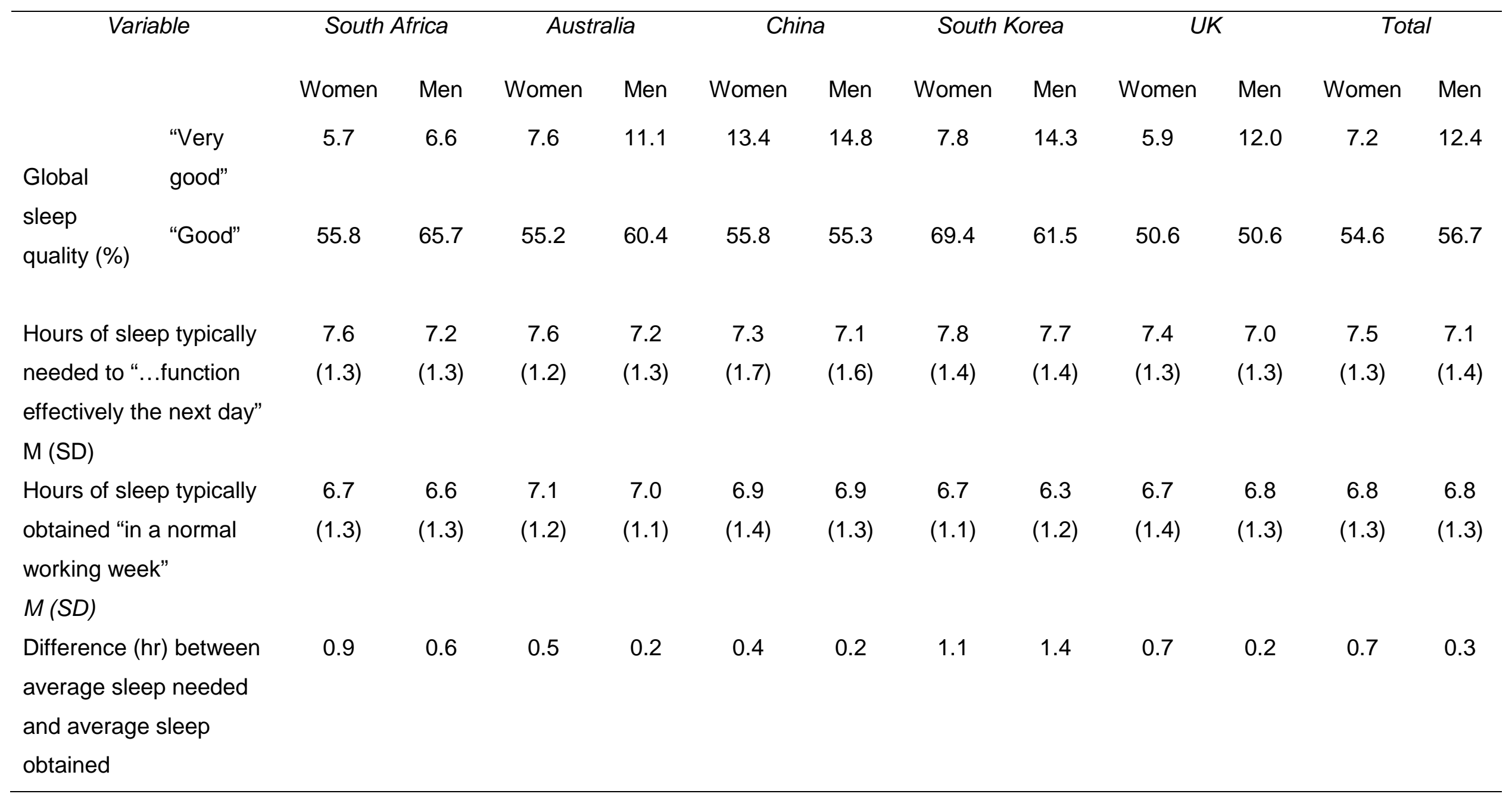


Table 3

Point prevalence of insomnia and levels of physical activity among survey participants in 5 countries

\begin{tabular}{|c|c|c|c|c|c|c|}
\hline Variable & South Africa & Australia & China & South Korea & UK & Total \\
\hline \multicolumn{7}{|c|}{ Prevalence of insomnia ${ }^{a}(95 \% \mathrm{Cl})$} \\
\hline Overall & $14.3 \%(12.8-15.7)$ & $10.7 \%(9.3-12.0)$ & $4.1 \%(3.1-5.0)$ & $6.5 \%(4.3-8.7)$ & $14.8 \%(13.5-16.1)$ & $11.5 \%(10.8-12.1)$ \\
\hline Women & $14.9 \%(13.3-16.4)$ & $11.9 \%(10.3-13.6)$ & $4.3 \%(3.0-5.7)$ & $6.9 \%(4.2-9.6)$ & $16.3 \%(14.8-17.8)$ & 13.0\% (12.3 -13.8) \\
\hline Men & $8.0 \%(4.2-11.8)$ & $7.2 \%(5.0-9.4)$ & $3.8 \%(2.4-5.1)$ & $5.6 \%(2.0-9.2)$ & $8.9 \%(6.6-11.3)$ & $6.5 \%(5.4-7.5)$ \\
\hline \multicolumn{7}{|l|}{ Overall } \\
\hline $\begin{array}{l}<10 \\
\mathrm{~min} / \text { week }^{\mathrm{b}}\end{array}$ & $8.0 \%(6.9-9.1)$ & $6.1 \%(5.1-7.1)$ & $6.0 \%(4.8-7.1)$ & $9.5 \%(6.9-12.1)$ & $7.2 \%(6.2-8.1)$ & $6.0 \%(5.5-6.5)$ \\
\hline $\begin{array}{l}10-75 \\
\mathrm{~min} / \text { week }^{\mathrm{b}}\end{array}$ & $56.9 \%(54.9-59.0)$ & $38.8 \%(36.7-41.0)$ & $40.4 \%(38.0-42.8)$ & $47.2 \%(42.8-51.6)$ & $34.1 \%(32.3-35.8)$ & $43.5 \%(42.5-44.6)$ \\
\hline $\begin{array}{l}150-300 \\
\text { min/week }\end{array}$ & $15.5 \%(14.1-17.0)$ & $24.5 \%(22.6-26.3)$ & $21.5 \%(19.5-23.5)$ & 19.6\% (16.1 -23.2) & $20.9 \%(19.4-22.4)$ & $20.4 \%(19.6-21.2)$ \\
\hline $\begin{array}{l}>300 \\
\mathrm{~min} / \text { week }\end{array}$ & $5.4 \%(4.5-6.4)$ & $11.7 \%(10.3-13.1)$ & $13.8 \%(12.2-15.5)$ & $10.9 \%(8.2-13.7)$ & $17.3 \%(15.9-18.7)$ & $12.2 \%(11.5-12.9)$ \\
\hline Women & & & & & & \\
\hline
\end{tabular}




\begin{tabular}{|c|c|c|c|c|c|c|}
\hline$<10$ & $8.1 \%(6.9-9.3)$ & $6.2 \%(5.0-7.4)$ & $6.1 \%(4.5-7.7)$ & $10.8 \%(7.5-14.2)$ & $7.4 \%(6.4-8.5)$ & $6.6 \%(6.1-7.2)$ \\
\hline \multicolumn{7}{|l|}{$\min /$ week $^{\mathrm{b}}$} \\
\hline $10-75$ & $57.7 \%(55.5$ - 59.8) & $41.4 \%(38.9-43.9)$ & $42.3 \%(39.0-45.6)$ & $47.1 \%(41.8-52.5)$ & $34.9 \%(33.0-36.9)$ & $45.2 \%(44.1-46.4)$ \\
\hline \multicolumn{7}{|l|}{$\mathrm{min} /$ week $^{\mathrm{b}}$} \\
\hline 76-149 & $14.5 \%(13.0-16.0)$ & $19.2 \%(17.2-21.2)$ & $18.3 \%(15.7-20.9)$ & $14.1 \%(10.4-17.9)$ & $21.2 \%(19.5-22.9)$ & $18.1 \%(17.2-19.0)$ \\
\hline \multicolumn{7}{|l|}{$\mathrm{min} /$ week $^{\mathrm{b}}$} \\
\hline $150-300$ & $14.6 \%(13.1-16.2)$ & $23.4 \%(21.2-25.6)$ & $20.5 \%(17.8-23.2)$ & $18.6 \%(14.4-22.8)$ & $20.5 \%(18.9-22.2)$ & $19.3 \%(18.4-20.3)$ \\
\hline \multicolumn{7}{|l|}{ min/week } \\
\hline$>300$ & $5.1 \%(4.2-6.1)$ & $9.8 \%(8.3-11.3)$ & $12.8 \%(10.5-15.0)$ & $9.3 \%(6.2-12.5)$ & $15.9 \%(14.4-17.4)$ & 10.7\% (10.0 -11.5) \\
\hline \multicolumn{7}{|l|}{ min/week } \\
\hline \multicolumn{7}{|l|}{ Men } \\
\hline$<10$ & $7.0 \%(3.4-10.6)$ & $5.7 \%(3.8-7.7)$ & $5.8 \%(4.1-7.5)$ & $6.8 \%(2.9-10.7)$ & $6.0 \%(4.1-8.0)$ & $4.1 \%(3.3-4.9)$ \\
\hline \multicolumn{7}{|l|}{$\min /$ week $^{b}$} \\
\hline $10-75$ & $49.0 \%(42.0-56.0)$ & $31.8 \%(27.9-35.7)$ & $38.2 \%(34.7-41.7)$ & $47.2 \%(39.4-55.0)$ & $30.8 \%(27.0-34.5)$ & $38.3 \%(36.2-40.3)$ \\
\hline \multicolumn{7}{|l|}{$\mathrm{min} /$ week $^{\mathrm{b}}$} \\
\hline 76-149 & $10.0 \%(5.8-14.2)$ & $18.1 \%(14.9-21.4)$ & $18.3 \% \%(15.5-21.9)$ & $9.9 \%(5.3-14.6)$ & $18.4 \%(15.2-21.5)$ & 16.9\% (15.4 -18.5) \\
\hline \multicolumn{7}{|l|}{$\min w_{e e k^{b}}$} \\
\hline $150-300$ & $25.0 \%(19.0-31.1)$ & $27.4 \%(23.6-31.1)$ & $22.6 \%(19.6-25.6)$ & $21.7 \%(15.3-28.2)$ & $22.3 \%(18.9-25.7)$ & $23.8 \%(22.1-25.6)$ \\
\hline \multicolumn{7}{|l|}{ min/week } \\
\hline$>300$ & $9.0 \%(5.0-13.0)$ & $17.0 \%(13.8-20.2)$ & $15.1 \%(12.5-17.7)$ & $14.3 \%(8.8-19.8)$ & $22.5 \%(19.1-25.9)$ & 16.9\% (15.3 -18.4) \\
\hline $\mathrm{min} /$ week & & & & & & \\
\hline
\end{tabular}

Note. $\mathrm{Cl}=$ confidence interval. 


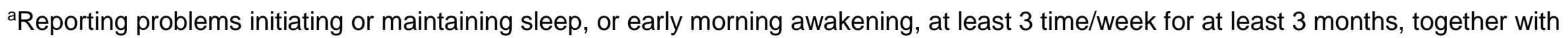
daytime dysfunction.

${ }^{b}$ Categories below World Health Organization (WHO, 2010) recommended minimum threshold of 150 mins/week of moderate intensity activity 
Table 4

Insomnia ${ }^{\mathrm{a}}$ risk associated with demographic, health and activity variables

\begin{tabular}{|c|c|c|c|c|c|c|}
\hline \multirow[b]{2}{*}{ Independent Variables } & \multicolumn{2}{|l|}{ Model 1} & \multicolumn{2}{|l|}{ Model 2} & \multicolumn{2}{|l|}{ Model 3} \\
\hline & OR $(95 \% \mathrm{Cl})$ & $\mathrm{p}$ & OR $(95 \% \mathrm{Cl})$ & $\mathrm{p}$ & OR $(95 \% \mathrm{Cl})$ & $\mathrm{p}$ \\
\hline \multicolumn{7}{|c|}{ Physical activity Level (reference $=76-149$} \\
\hline \multicolumn{7}{|l|}{$\min /$ week) } \\
\hline$<10 \mathrm{~min} /$ day & $1.37(1.05-1.79)$ & .015 & $1.06(0.80-1.40)$ & .694 & $1.07(0.81-1.42)$ & .638 \\
\hline 10-75 min/week & $1.11(0.93-1.34)$ & .264 & $0.98(0.81-1.18)$ & .801 & $0.98(0.80-1.19)$ & .842 \\
\hline 150-300 min/week & $0.98(0.79-1.22)$ & .839 & $1.06(0.85-1.33)$ & .589 & $1.08(0.86-1.35)$ & .531 \\
\hline$>300 \mathrm{~min} /$ week & $1.20(0.94-1.53)$ & .138 & $1.30(1.01-1.65)$ & .045 & $1.31(1.02-1.69)$ & .033 \\
\hline \multicolumn{7}{|l|}{ Gender (reference $=$ Male) } \\
\hline Female & $2.09(1.74-2.52)$ & $<.001$ & $2.02(1.67-2.45)$ & $<.001$ & $1.64(1.35-2.00)$ & $<.001$ \\
\hline \multicolumn{7}{|l|}{ Age $($ reference $=18-34)$} \\
\hline $35-54$ & $1.50(1.28-1.75)$ & $<.001$ & $1.36(1.16-1.60)$ & $<.001$ & $1.19(1.01-1.40)$ & .038 \\
\hline $55-64$ & $1.78(1.45-2.17)$ & $<.001$ & $1.55(1.26-1.91)$ & $<.001$ & $1.20(0.97-1.49)$ & .093 \\
\hline $65+$ & $1.47(1.09-1.98)$ & .012 & $1.13(0.82-1.55)$ & .453 & $0.85(0.62-1.17)$ & .315 \\
\hline \multicolumn{7}{|l|}{ Subjectively rated health (reference $=$} \\
\hline \multicolumn{7}{|l|}{ Average) } \\
\hline Below Average & & & $1.85(1.57-2.18)$ & $<.001$ & $1.76(1.49-2.07)$ & $<.001$ \\
\hline Above average & & & $0.74(0.63-0.88)$ & $<.001$ & $0.70(0.59-0.83)$ & $<.001$ \\
\hline
\end{tabular}


Sleep medication use in previous 3 months

(reference $=$ No)

Yes

Smoking status (reference $=$ Non Smoker)

Smoker

Alcohol Consumption (reference $<21$

units/week)

$>21$ units/week

Usually consumes caffeinated drink within 1

hour of going to bed (reference $=\mathrm{No}$ )

Yes

Educational level (reference $=$ High)

Medium

Low

Employment status (reference $=$ Not

formally employed)

Formally employed

Country or residence (reference $=$ UK)

South Africa

Australia

China

South Korea

\section{$3.75(3.08-4.57)$}

$1.27(1.06-1.52)$

.010

$1.22(1.02-1.46)$

.033

$1.11(0.88-1.39)$

$1.11(0.88-1.39)$

$$
0.92(0.75-1.12)
$$

$1.55(1.12-2.15)$

$1.30(1.11-1.53)$

.008

.001

$1.43(1.03-1.98)$

$1.21(1.03-1.43)$

.034

.021

$0.87(0.75-1.01)$

.058

$0.79(0.68-0.92)$

.003

$0.84(0.670-1.01)$

.063

$1.02(0.85-1.22)$

.839

$0.33(0.24-0.44)$

$<.001$

$0.45(0.31-0.67)$ 


\begin{tabular}{lcc}
\hline Interaction terms & $\mathrm{p}$ & $\mathrm{p}$ \\
\hline Physical activity level X Gender & .804 & .863 \\
Physical activity level X Employment status & .606 & .781 \\
Country X Physical activity level & & .549 \\
Country X Employment status & .266 \\
Country X Gender & .098 \\
Country X Age & .576 \\
\hline
\end{tabular}

Note. OR = odds ratio $; \mathrm{Cl}=95 \%$ confidence interval.

${ }^{a}$ Reporting problems initiating or maintaining sleep, or early morning awakening, at least 3 time/week for at least 3 months, together with daytime dysfunction. 


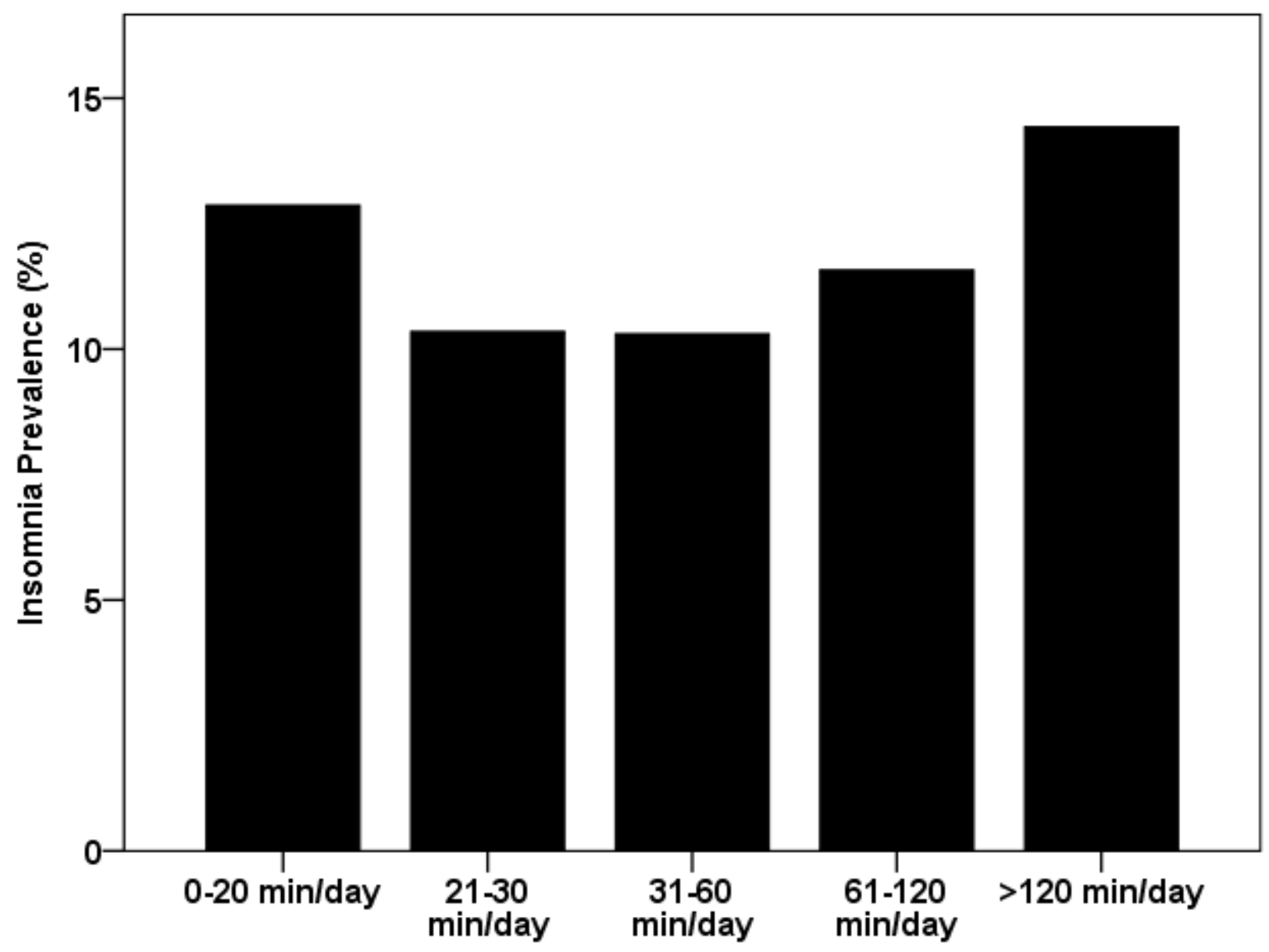

FIGURE 1 Insomnia prevalence associated with the typical duration of moderate intensity physical activity (performed in bouts of $\geq 10$ continuous minutes) per active day. Note.

Insomnia case/non-case association with duration of physical activity $X^{2}(4,9238)=14.77$, $\mathrm{p}<.005)$. 


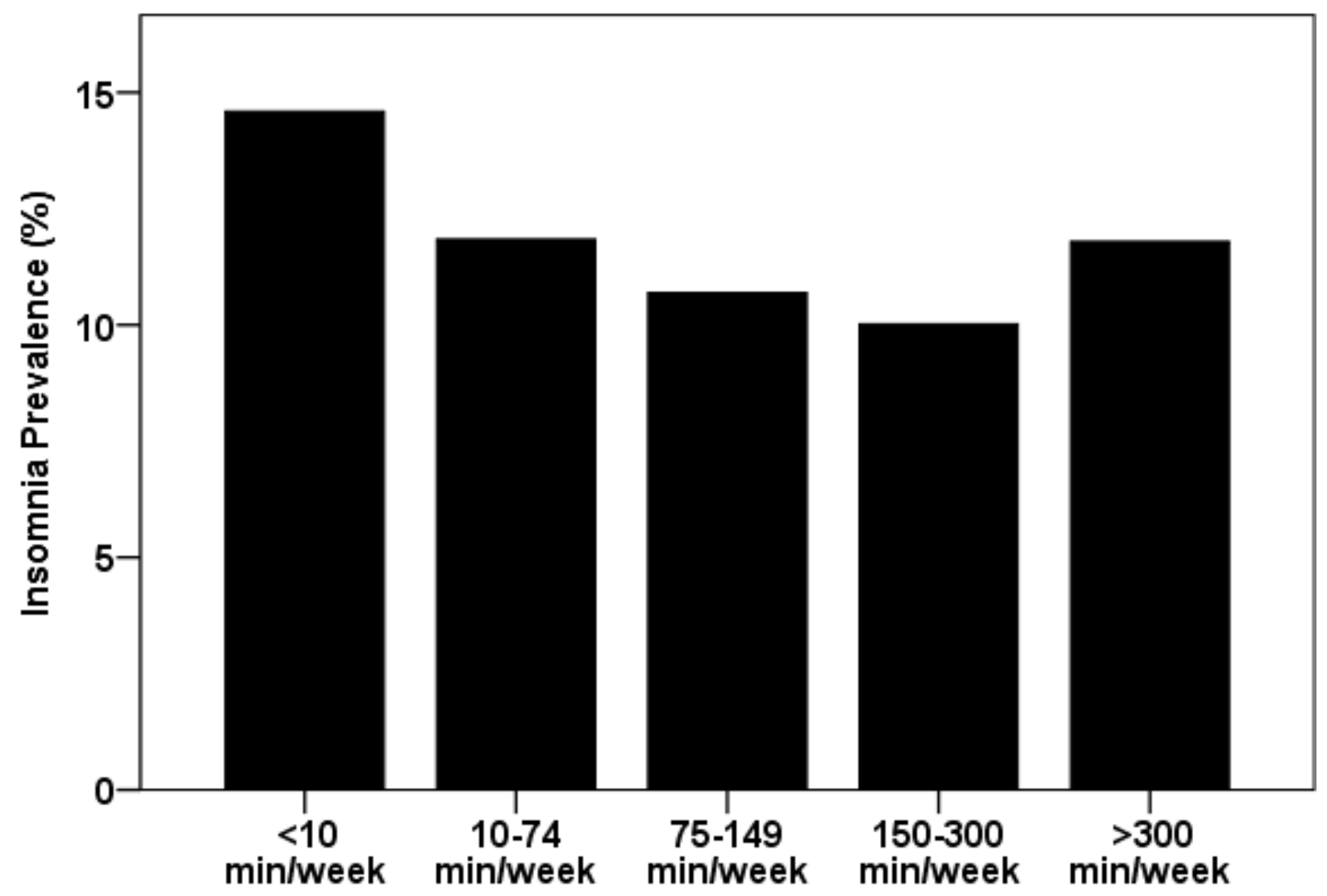

FIGURE 2 Insomnia prevalence associated with typical weekly duration (range) of moderate intensity physical activity (performed in bouts of $\geq 10$ continuous minutes). Note. Insomnia case/non-case association with weekly duration of physical activity: $X^{2}(4,9238)=$ $14.90, \mathrm{p}<.01)$. 
PHYSICAL ACTIVITY AND INSOMNIA 